\title{
Supracervical Hysterectomy
}

National Cancer Institute

\section{Source}

National Cancer Institute. Supracervical Hysterectomy. NCI Thesaurus. Code C126397.

A hysterectomy that removes the uterus and leaves the cervix in place. 